EUROPEAN JOURNAL OF PURE AND APPLIED MATHEMATICS

Vol. 12, No. 2, 2019, 590-604

ISSN 1307-5543 - www.ejpam.com

Published by New York Business Global

\title{
On a nonsingular equation of length 9 over torsion free groups
}

\author{
M. Fazeel Anwar ${ }^{1, *}$, Mairaj Bibi², M. Saeed Akram ${ }^{3}$ \\ 1 Department of Mathematics, Sukkur IBA University, Sukkur. \\ 2 Department of Mathematics, COMSATS Institute of Information Technology, Islamabad. \\ 3 Department of Mathematics, Khwaja Fareed University of Engineering 8 Information \\ Technology, Rahim Yar Khan.
}

\begin{abstract}
In [11], Levin conjectured that every equation is solvable over a torsion free group. In this paper we consider a nonsingular equation $g_{1} t g_{2} t g_{3} t g_{4} t g_{5} t g_{6} t^{-1} g_{7} t g_{8} t g_{9} t^{-1}=1$ of length 9 and show that it is solvable over torsion free groups modulo some exceptional cases.
\end{abstract}

2010 Mathematics Subject Classifications: 20F05, 57M05

Key Words and Phrases: Asphericity; relative group presentations; torsion-free groups; group equations.

\section{Introduction}

Let $G$ be a non-trivial group, $t$ be an unknown and let $F$ be a free group generated by $t$. An equation in $t$ over $G$ is an expression of the form

$$
s(t)=g_{1} t^{\epsilon_{1}} g_{2} t^{\epsilon_{2}} \cdots g_{n} t^{\epsilon_{n}}=1\left(g_{i} \in G, \epsilon_{i}= \pm 1\right)
$$

in which it is assumed that $\epsilon_{i}+\epsilon_{i+1}=0$ implies $g_{i+1} \neq 1$ in $G$. We call the integer $n$ the length of the equation. A solution of $s(t)=1$ over $G$ is an embedding $\phi$ of $G$ into a group $H$ and an element $h \in H$ such that $\phi\left(g_{1}\right) h^{\epsilon_{1}} \phi\left(g_{2}\right) h^{\epsilon_{2}} \cdots \phi\left(g_{n}\right) h^{\epsilon_{1}}=1$ in $H$. Equivalently $s(t)=1$ is solvable over $G$ if and only if the natural map from $G$ to $\langle G * F \mid s(t)\rangle$ is injective, where $G * F$ is the free product of $G$ and $F$. If $G$ is a torsion free group then by Levin's conjecture every equation is solvable [11]. The conjecture is known to be true for $n \leq 7$ $[5,7,9,12]$. The authors have done significant work in $[1,2,4]$ to establish the conjecture for $n=8$.

The equation of length 9 have been consider in [3]. It has been proved that there are only three equations of length 9 which are still open. In this paper we consider a nonsingular equation of length 9 (one of three) and show that the equation has a solution

* Corresponding author.

DOI: https://doi.org/10.29020/nybg.ejpam.v12i2.3405

Email addresses: fazeel.anwar@iba-suk.edu.pk (M. Fazeel Anwar) 
over $G$ modulo some exceptional cases. This paper is the first step in proving Levin's conjecture for equations of length 9 .

We first give some basic definitions. A relative group presentation is a presentation of the form $\mathcal{P}=\langle G, x \mid r\rangle$ where $r$ is a set of cyclically reduced words in $G *\langle x\rangle$. If the relative presentation is orientable and aspherical then the natural map from $G$ to $\langle G, x \mid r\rangle$ is injective. In our case $x$ and $r$ consist of the single element $t$ and $s(t)$ respectively, therefore $\mathcal{P}$ is orientable and so asphericity implies $s(t)=1$ is solvable. In this paper we use the weight test and the curvature distribution method to show that $\mathcal{P}$ is aspherical [6].

The star graph $\Gamma$ of $\mathcal{P}$ has vertex set $x \cup x^{-1}$ and edge set $r^{*}$, where $r^{*}$ is the set of all cyclic permutations of the elements of $r \cup r^{-1}$ which begin with an element of $x \cup x^{-1}$. For $R \in r^{*}$ write $R=S g$ where $g \in G$ and $S$ begins and ends with $x$ symbols. Then $\mathfrak{i}(R)$ is the inverse of the last symbol of $S, \tau(R)$ the first symbol of $S$ and $\lambda(R)=g$. A weight function $\theta$ on $\Gamma$ is a real valued function on the set of edges of $\Gamma$ which satisfies $\theta(S h)=\theta\left(S^{-1} h^{-1}\right)$. A weight function $\theta$ is called aspherical if the following three conditions are satisfied

(W1) Let $R \in r^{*}$ with $R=x_{1}^{\epsilon_{1}} g_{1} \cdots x_{n}^{\epsilon_{n}} g_{n}$. Then

$$
\sum_{i=1}^{n}\left(1-\theta\left(x_{i}^{\epsilon_{i}} g_{i} \cdots x_{n}^{\epsilon_{n}} g_{n} x_{1}^{\epsilon_{1}} g_{1} \cdots x_{i-1}^{\epsilon_{i-1}} g_{i-1}\right)\right) \geq 2 .
$$

(W2) Each admissible cycle in $\Gamma$ has weight at least 2 (where admissible means having a label trivial in $G$ ).

(W3) Each edge of $\Gamma$ has a non-negative weight.

If $\Gamma$ admits an aspherical weight function then $\mathcal{P}$ is aspherical [6]. The following lemma [10] tells us that we can apply asphericity test in $k$-steps.

Lemma 1. Let the relative presentation $P=\langle H, x: r\rangle$ define a group $G$ and let $Q=$ $\langle G, t: s\rangle$ be another relative presentation. If $Q$ and $P$ are both aspherical, then the relative presentation $R=\langle H, x \cup t: r \cup \tilde{s}\rangle$ is aspherical, where $\tilde{s}$ is an element of $H * F(x) * F(t)$ obtained from s by lifting.

For a detailed account on the curvature distribution method see [3]. It is clear from our definition of a group equation that if $g_{i}$ is a coefficient between a negative and a positive power of $t$ than $g_{i}$ is not trivial in $G$. This fact will be used in all subsequent proofs without reference.

\section{Main Results}

We now turn our attention to length 9 equations. A list of these equations is given in [3]. Consider the nonsingular equation of length 9 given by atbtctdtet ft ${ }^{-1}$ gthtit $^{-1}=1$. We write this as $\mathcal{P}=\langle G, t \mid s(t)\rangle$, where $s(t)=$ atbtctdtet $f t^{-1}$ gthtit $^{-1}$.

Here $b, c, d, e, h \in G$ and $a, f, g, i \in G \backslash\{1\}$. The star graph $\Gamma$ for $\mathcal{P}$ is given in Figure 2. We apply the transformation $x=t b$ to get that $b=1$ in $G$. By using the methods 


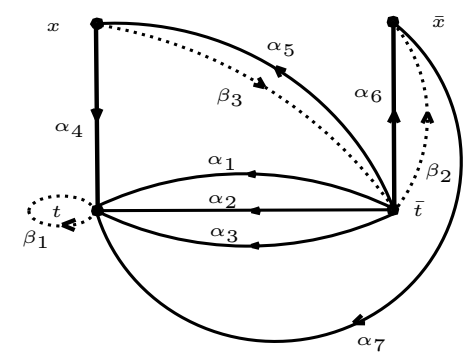

(a)

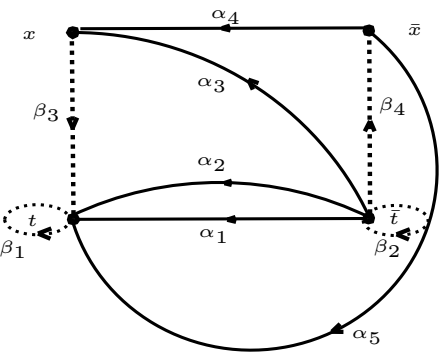

(b)

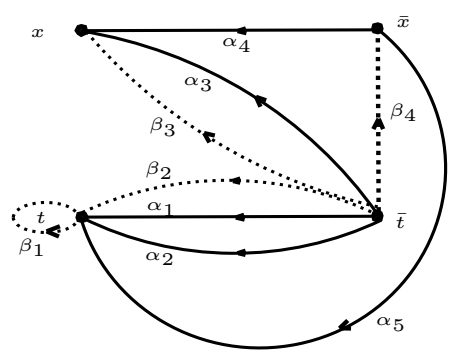

(c)

Figure 1: Star graph $\Gamma$

given in $[5,8]$ we conclude that possible vertices of degree 2 (in the diagram associated to $\mathcal{P})$ are (upto cyclic permutation and inversion)

$$
S=\left\{a g, a g^{-1}, f i, f i^{-1}, b c^{-1}, b d^{-1} b e^{-1}, b h^{-1}, c d^{-1}, c e^{-1}, c h^{-1}, d e^{-1}, d h^{-1}, e h^{-1}\right\} .
$$

Since $G$ is torsion free therefore it is clear that $a g$ and $a g^{-1}$ can not both hold at the same time. Similarly $f i$ and $f i^{-1}$ can not both hold at the same time. The following lemma gives some general results that will greatly simplify the proofs. This is an application of the results given in $[1,2]$.

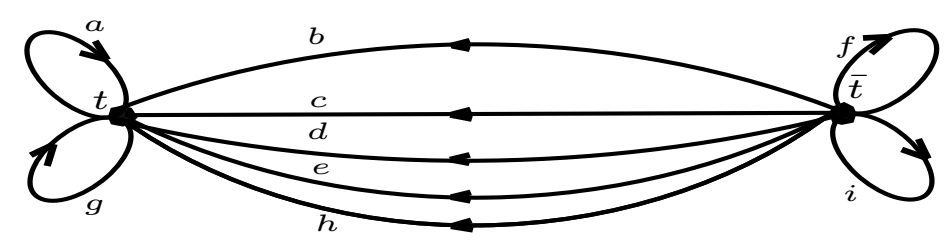

Figure 2: Star graph $\Gamma$

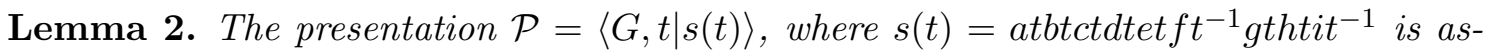
pherical if any one of the following holds:

(i) $a=g^{-1}$

(ii) $a=g, f=i$ 
(iii) $a=g, b=h$

Proof. A new generator $x$ will be introduced to obtain the presentation $\mathcal{Q}=\left\langle G, t, x \mid r_{1}, r_{2}\right\rangle$.

(i) Let $a=g^{-1}$. The relator $s(t)$ is given by $s(t)=$ atbtctdtetf $t^{-1} a^{-1}$ thtit $^{-1}$. We substitute $x=t^{-1} a^{-1} t$ to get $r_{1}=x^{-1}$ btctdtet fxhti and $r_{2}=t^{-1} a^{-1} t x^{-1}$. The star graph $\Gamma$ for $\mathcal{Q}$ is given by Figure 1 (a) in which (using $r_{1}$ ) $\alpha_{1}=e, \alpha_{2}=d, \alpha_{3}=$ $c, \alpha_{4}=b, \alpha_{5}=f, \alpha_{6}=i, \alpha_{7}=h$; and (using $\left.r_{2}\right) \beta_{1}=a^{-1}, \beta_{2}=1, \beta_{3}=1$. We assign a weight function $\theta$ such that $\theta\left(\alpha_{4}\right)=\theta\left(\alpha_{6}\right)=\theta\left(\beta_{1}\right)=\theta\left(\beta_{2}\right)=0$. All other edges are assigned a weight 1 . Then $\Sigma\left(1-\theta\left(\alpha_{i}\right)\right)=\Sigma\left(1-\theta\left(\beta_{j}\right)\right)=2$ shows that (W1) is satisfied. Also each cycle in $\Gamma$ of weight less than 2 has label $a^{m}$ or $i^{m}$, $(m \neq 0)$ and $(a, i \neq 1)$ and since $G$ is torsion free (W2) is satisfied. Moreover (W3) clearly holds.

(ii) We have

$$
s(t)=\text { atbtctdtetit }^{-1} a t h t i t^{-1}, r_{1}=x b t c t d t e x h, r_{2}=t i t^{-1} a t x^{-1} .
$$

The star graph $\Gamma$ is given by Figure 1 (b) in which $\alpha_{1}=c, \alpha_{2}=d, \alpha_{3}=e, \alpha_{4}=$ $h, \alpha_{5}=b ;$ and $\beta_{1}=a, \beta_{2}=i, \beta_{3}=1, \beta_{4}=1$. We assign a weight function $\theta$ such that $\theta\left(\alpha_{3}\right)=\theta\left(\alpha_{5}\right)=\theta\left(\beta_{1}\right)=\theta\left(\beta_{2}\right)=0$. All other edges are assigned a weight 1 . Then $\Sigma\left(1-\theta\left(\alpha_{i}\right)\right)=\Sigma\left(1-\theta\left(\beta_{j}\right)\right)=2$ shows that (W1) is satisfied. Also each cycle in $\Gamma$ of weight less than 2 has label $a^{m}$ or $i^{m},(m \neq 0)$ and $(a, i \neq 1)$ and since $G$ is torsion free (W2) is satisfied. Moreover (W3) clearly holds.

(iii) We have

$$
s(t)=\text { atbtctdtetf } t^{-1} a t b t i t^{-1}, r_{1}=x c t d t e t f x i, r_{2}=t^{-1} a t b t x^{-1} .
$$

The star graph $\Gamma$ is given by Figure 1 (c) in which $\alpha_{1}=e, \alpha_{2}=d, \alpha_{3}=f, \alpha_{4}=$ $i, \alpha_{5}=c$; and $\beta_{1}=a, \beta_{2}=b, \beta_{3}=1, \beta_{4}=1$. We assign a weight function $\theta$ such that $\theta\left(\alpha_{3}\right)=\theta\left(\alpha_{5}\right)=\theta\left(\beta_{1}\right)=\theta\left(\beta_{3}\right)$. All other edges are assigned a weight 1 .

We have the desired result.

Corollary 1. The presentation $\mathcal{P}=\langle G, t \mid s(t)\rangle$, is aspherical if any one of the following holds:

(i) $a=g^{-1}$ and $R \in\left\{f i, f i^{-1}, b c^{-1}, b d^{-1} b e^{-1}, b h^{-1}, c d^{-1}, c e^{-1}, c h^{-1}, d e^{-1}, d h^{-1}, e h^{-1}\right\}$

(ii) $a=g, f=i$ and $R \in\left\{b c^{-1}, b d^{-1} b e^{-1}, b h^{-1}, c d^{-1}, c e^{-1}, c h^{-1}, d e^{-1}, d h^{-1}, e h^{-1}\right\}$

(iii) $a=g, b=h$ and $R \in\left\{c d^{-1}, c e^{-1}, c h^{-1}, d e^{-1}, d h^{-1}, e h^{-1}\right\}$

Proof. The result is clear from lemma 3 by taking the weight function as given in lemma 3, part 1, 2 and 3 respectively. 
M. Fazeel Anwar, Mairaj Bibi, M. Saeed Akram / Eur. J. Pure Appl. Math, 12 (2) (2019), 590-604 594


pherical if any one of the following holds:

(i) $a=g, b=c$

(ii) $a=g, b=d$

(iii) $a=g, b=e$

(iv) $a=g, c=d$

(v) $a=g, c=e$

(vi) $a=g, c=h$

(vii) $a=g, d=e$

(viii) $a=g, d=h$

(ix) $a=g, e=h$

(x) $b=c, d=h$

(xi) $b=c, e=h$

(xii) $b=d, c=e$

(xiii) $b=d, c=h$

(xiv) $b=d, e=h$

(xv) $b=e, c=h$

(xvi) $b=h, c=d$

(xvii) $b=c, d=e$

Proof.

(i) In this case $\Delta$ is shown in Figure 3. Since $d_{\Delta}\left(v_{a}\right)=d_{\Delta}\left(v_{b}\right)=2$ or $d_{\Delta}\left(v_{b}\right)=d_{\Delta}\left(v_{c}\right)=$ 2 can not occur therefore it can be assumed that $d_{\Delta}\left(v_{a}\right)=d_{\Delta}\left(v_{c}\right)=d_{\Delta}\left(v_{g}\right)=2$ as shown in Figure 3. In this case $c(\Delta) \leq 0$. 

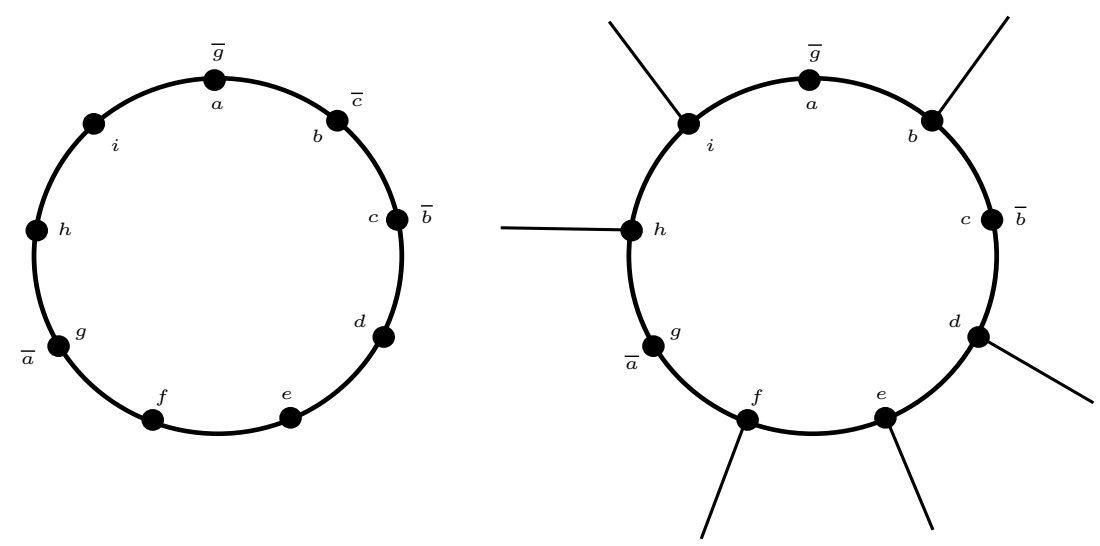

Figure 3: Region $\Delta$

(ii) In this case $\Delta$ is shown in Figure 4 . Since degree of vertices $v_{a}$ and $v_{b}$ can not be 2 together so there are the following two cases to consider:

(a) $d_{\Delta}\left(v_{a}\right)=d_{\Delta}\left(v_{d}\right)=d_{\Delta}\left(v_{g}\right)=2$;

(b) $d_{\Delta}\left(v_{b}\right)=d_{\Delta}\left(v_{d}\right)=d_{\Delta}\left(v_{g}\right)=2$.

as shown in Figure 4. In both of these cases $c(\Delta) \leq 0$.


Figure 4: Region $\Delta$

(iii) In this case $\Delta$ is shown in Figure 5. Since degree of vertices $v_{a}$ and $v_{b}$ can not be 2 together so there are the following two cases to consider: 
M. Fazeel Anwar, Mairaj Bibi, M. Saeed Akram / Eur. J. Pure Appl. Math, 12 (2) (2019), 590-604 596

(a) $d_{\Delta}\left(v_{a}\right)=d_{\Delta}\left(v_{e}\right)=d_{\Delta}\left(v_{g}\right)=2$;

(b) $d_{\Delta}\left(v_{b}\right)=d_{\Delta}\left(v_{e}\right)=d_{\Delta}\left(v_{g}\right)=2$.

as shown in Figure 5. In both of these cases $c(\Delta) \leq 0$.
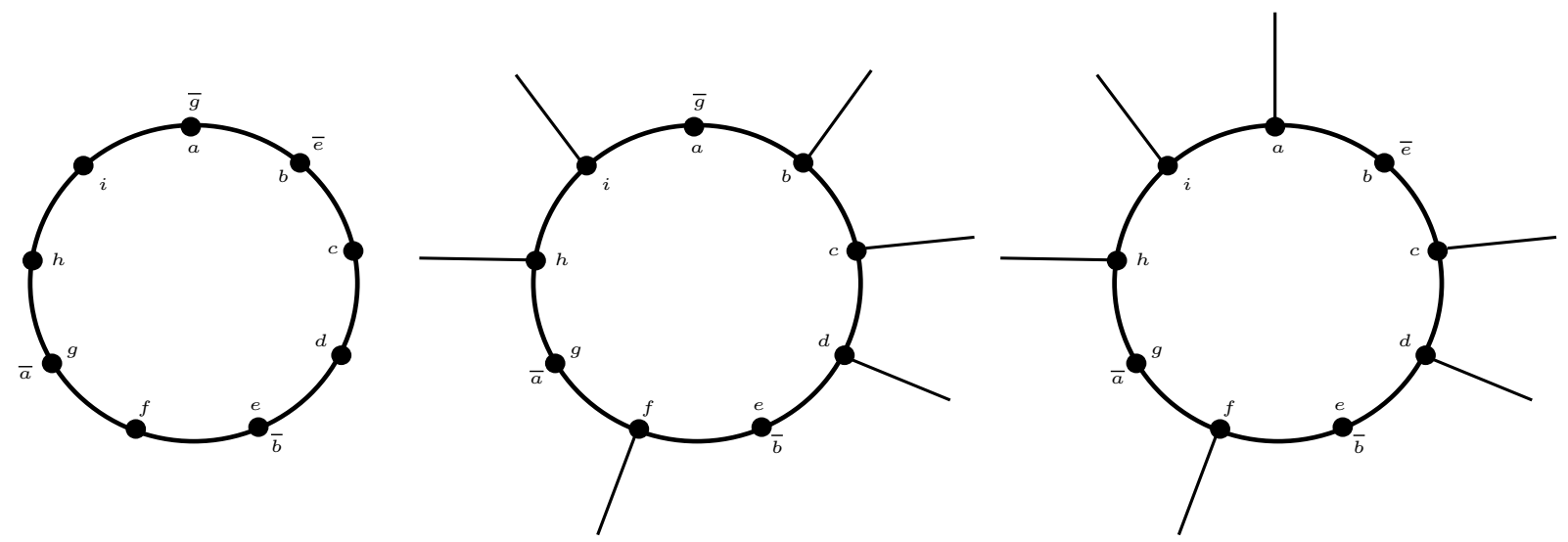

Figure 5: Region $\Delta$

(iv) In this case $\Delta$ is shown in Figure 6 . Since degree of vertices $v_{a}$ and $v_{b}$ can not be 2 together so there are the following two cases to consider:

(a) $d_{\Delta}\left(v_{a}\right)=d_{\Delta}\left(v_{c}\right)=d_{\Delta}\left(v_{g}\right)=2$;

(b) $d_{\Delta}\left(v_{a}\right)=d_{\Delta}\left(v_{d}\right)=d_{\Delta}\left(v_{g}\right)=2$.

as shown in Figure 6. In both of these cases $c(\Delta) \leq 0$. 



Figure 6: Region $\Delta$

(v) In this case $\Delta$ is shown in Figure 7. Here $d_{\Delta}\left(v_{a}\right)=d_{\Delta}\left(v_{c}\right)=d_{\Delta}\left(v_{e}\right)=d_{\Delta}\left(v_{g}\right)=2$ which implies $l_{\Delta}\left(v_{a}\right)=a g^{-1}, l_{\Delta}\left(v_{c}\right)=c e^{-1}, l_{\Delta}\left(v_{e}\right)=e c^{-1}$ ans $l_{\Delta}\left(v_{g}\right)=g a^{-1}$ as shown in Figure 7 . In order to have positive curvature the remaining vertices must be of degree 3. Observe that $l_{\Delta}\left(v_{a}\right)=a g^{-1}$ and $l_{\Delta}\left(v_{c}\right)=c e^{-1}$ implies that $l_{\Delta}\left(v_{b}\right)=$ $h^{-1} b d^{-1} w$ where $w \in\{b, c, d, e, h\}$ which implies $d_{\Delta}\left(v_{b}\right)>3$. Notice that $l_{\Delta}\left(v_{e}\right)=$ $e c^{-1}$ and $l_{\Delta}\left(v_{g}\right)=g a^{-1}$ implies that $l_{\Delta}\left(v_{f}\right)=d^{-1} f i^{-1} w$ where $w \in\{b, c, d, e, h\}$ which implies $d_{\Delta}\left(v_{f}\right)>3$. Since $d_{\Delta}\left(v_{b}\right)>3$ and $d_{\Delta}\left(v_{f}\right)>3$ so $c(\Delta) \leq 0$.
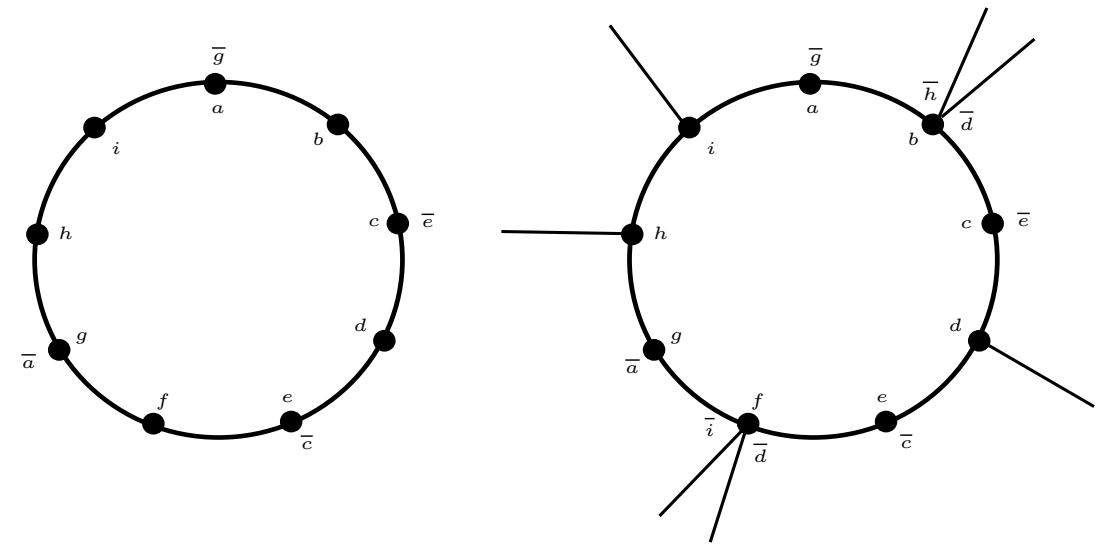

Figure 7: Region $\Delta$

(vi) In this case $\Delta$ is shown in Figure 8. Since degree of vertices $v_{g}$ and $v_{h}$ can not be 2 together so there are the following two cases to consider: 
(a) $d_{\Delta}\left(v_{a}\right)=d_{\Delta}\left(v_{c}\right)=d_{\Delta}\left(v_{g}\right)=2$;

(b) $d_{\Delta}\left(v_{a}\right)=d_{\Delta}\left(v_{c}\right)=d_{\Delta}\left(v_{h}\right)=2$.

as shown in Figure 8. In both of these cases $c(\Delta) \leq 0$.
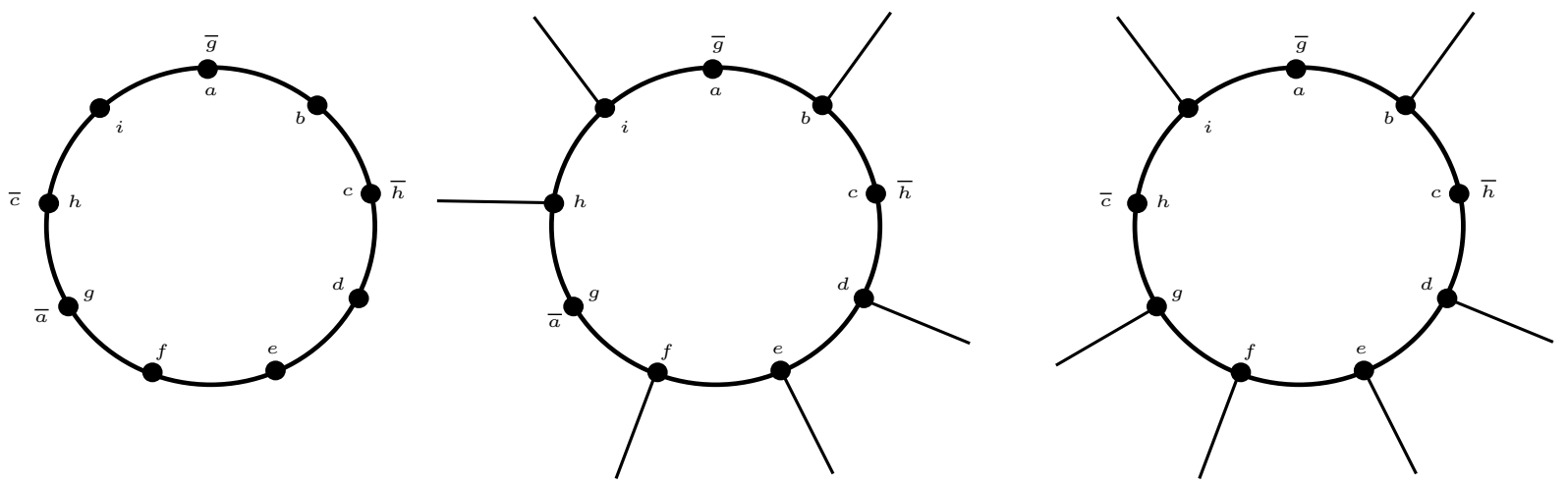

Figure 8: Region $\Delta$

(vii) In this case $\Delta$ is shown in Figure 9. Since degree of vertices $v_{d}$ and $v_{e}$ can not be 2 together so there are the following two cases to consider:

(a) $d_{\Delta}\left(v_{a}\right)=d_{\Delta}\left(v_{d}\right)=d_{\Delta}\left(v_{g}\right)=2$;

(b) $d_{\Delta}\left(v_{a}\right)=d_{\Delta}\left(v_{e}\right)=d_{\Delta}\left(v_{g}\right)=2$.

as shown in Figure 9. In both of these cases $c(\Delta) \leq 0$.
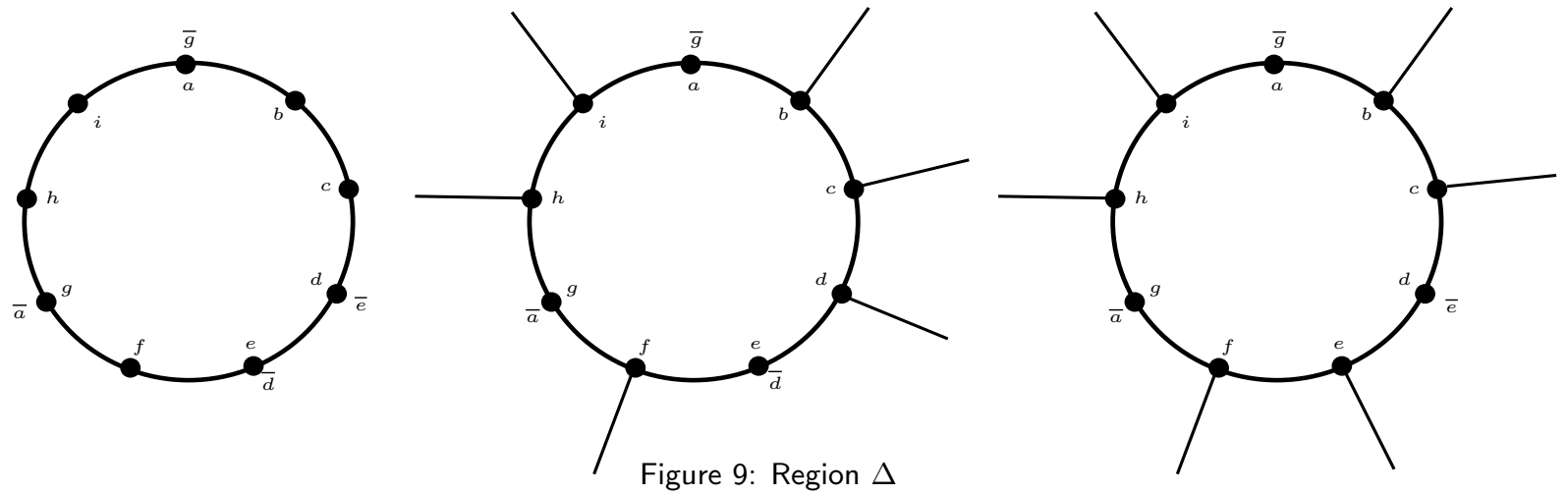

(viii) In this case $\Delta$ is shown in Figure 10. Since degree of vertices $v_{g}$ and $v_{h}$ can not be 2 together so there are the following two cases to consider:

(a) $d_{\Delta}\left(v_{a}\right)=d_{\Delta}\left(v_{d}\right)=d_{\Delta}\left(v_{g}\right)=2 ;$ 
(b) $d_{\Delta}\left(v_{a}\right)=d_{\Delta}\left(v_{d}\right)=d_{\Delta}\left(v_{h}\right)=2$.

as shown in Figure 10. In both of these cases $c(\Delta) \leq 0$.
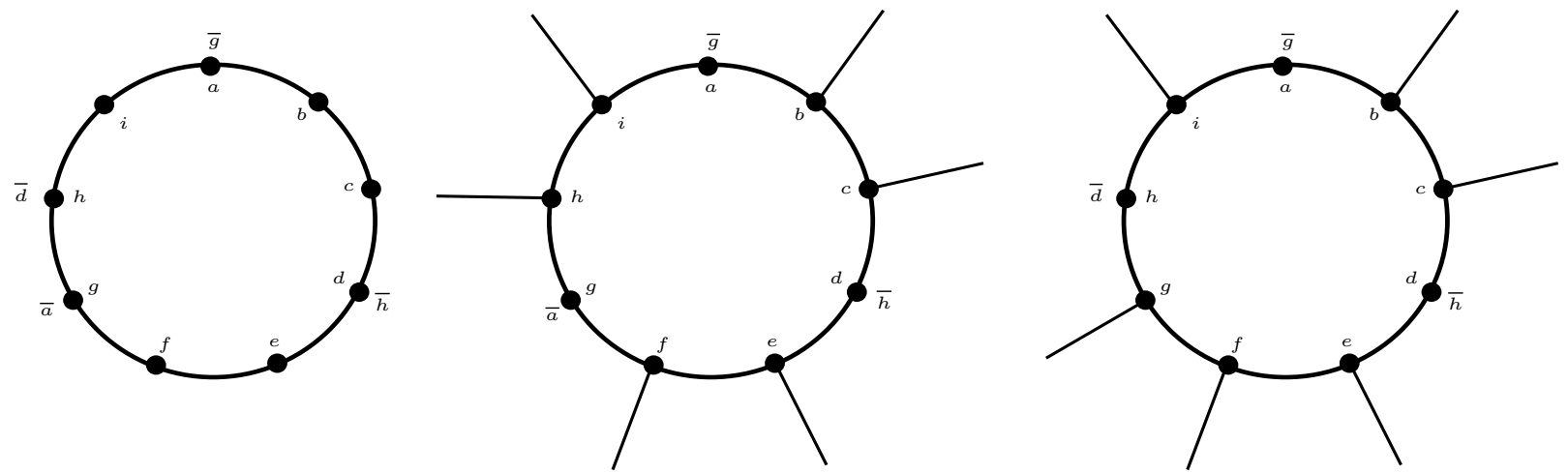

Figure 10: Region $\Delta$

(ix) In this case $\Delta$ is shown in Figure 11. Since degree of vertices $v_{g}$ and $v_{h}$ can not be 2 together so there are the following two cases to consider:

(a) $d_{\Delta}\left(v_{a}\right)=d_{\Delta}\left(v_{e}\right)=d_{\Delta}\left(v_{g}\right)=2$;

(b) $d_{\Delta}\left(v_{a}\right)=d_{\Delta}\left(v_{e}\right)=d_{\Delta}\left(v_{h}\right)=2$.

as shown in Figure 11. In both of these cases $g(\Delta) \leq 0$.
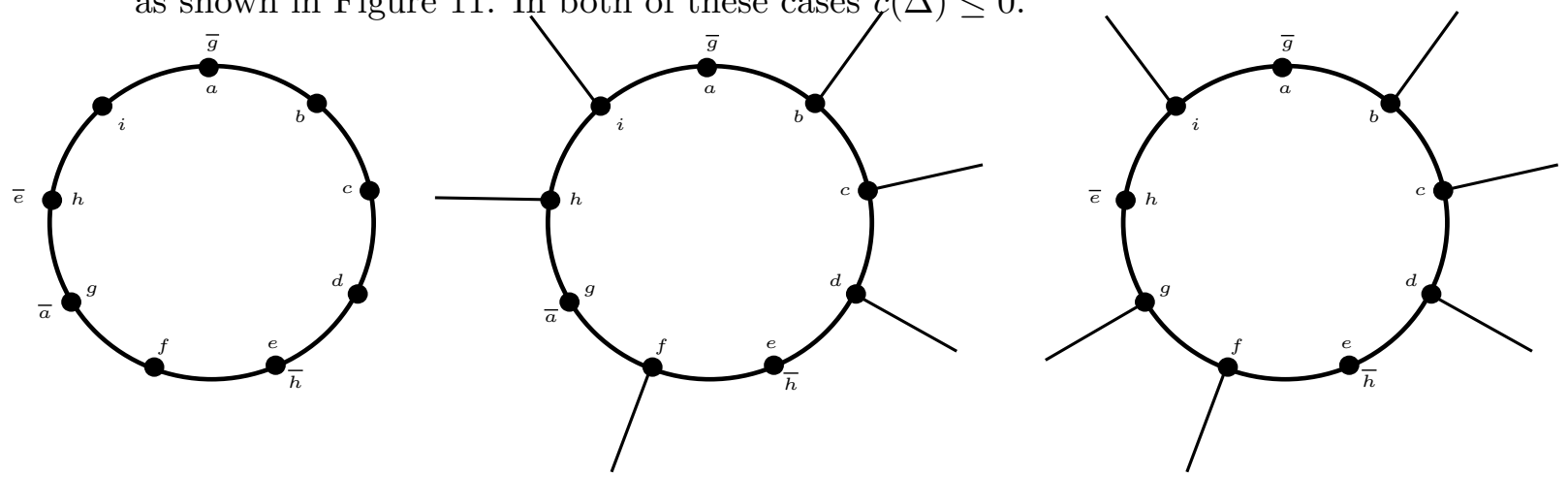

Figure 11: Region $\Delta$

(x) In this case $\Delta$ is shown in Figure 12. Since $d_{\Delta}\left(v_{b}\right)=d_{\Delta}\left(v_{c}\right)=2$ or $d_{\Delta}\left(v_{c}\right)=$ $d_{\Delta}\left(v_{d}\right)=2$ can not occur therefore it can be assumed that $d_{\Delta}\left(v_{b}\right)=d_{\Delta}\left(v_{d}\right)=$ $d_{\Delta}\left(v_{h}\right)=2$ as shown in Figure 12. In this case $c(\Delta) \leq 0$. 

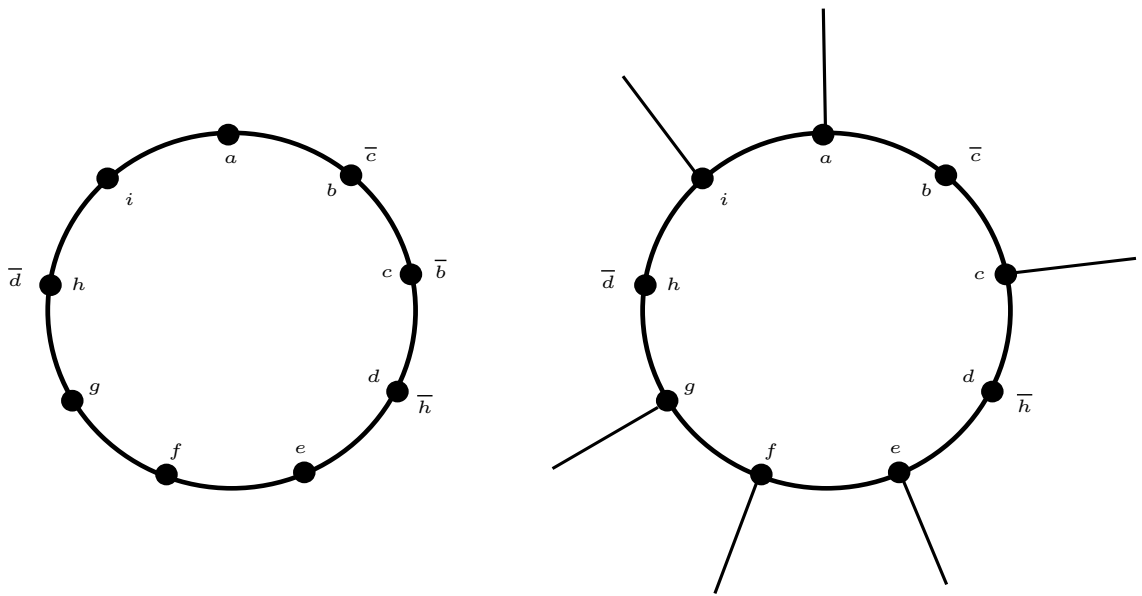

Figure 12: Region $\Delta$

(xi) In this case $\Delta$ is shown in Figure 13. Since degree of vertices $v_{b}$ and $v_{c}$ can not be 2 together so there are the following two cases to consider:

(a) $d_{\Delta}\left(v_{b}\right)=d_{\Delta}\left(v_{e}\right)=d_{\Delta}\left(v_{h}\right)=2$;

(b) $d_{\Delta}\left(v_{c}\right)=d_{\Delta}\left(v_{e}\right)=d_{\Delta}\left(v_{h}\right)=2$.

as shown in Figure 13. In both of these cases $c(\Delta) \leq 0$.
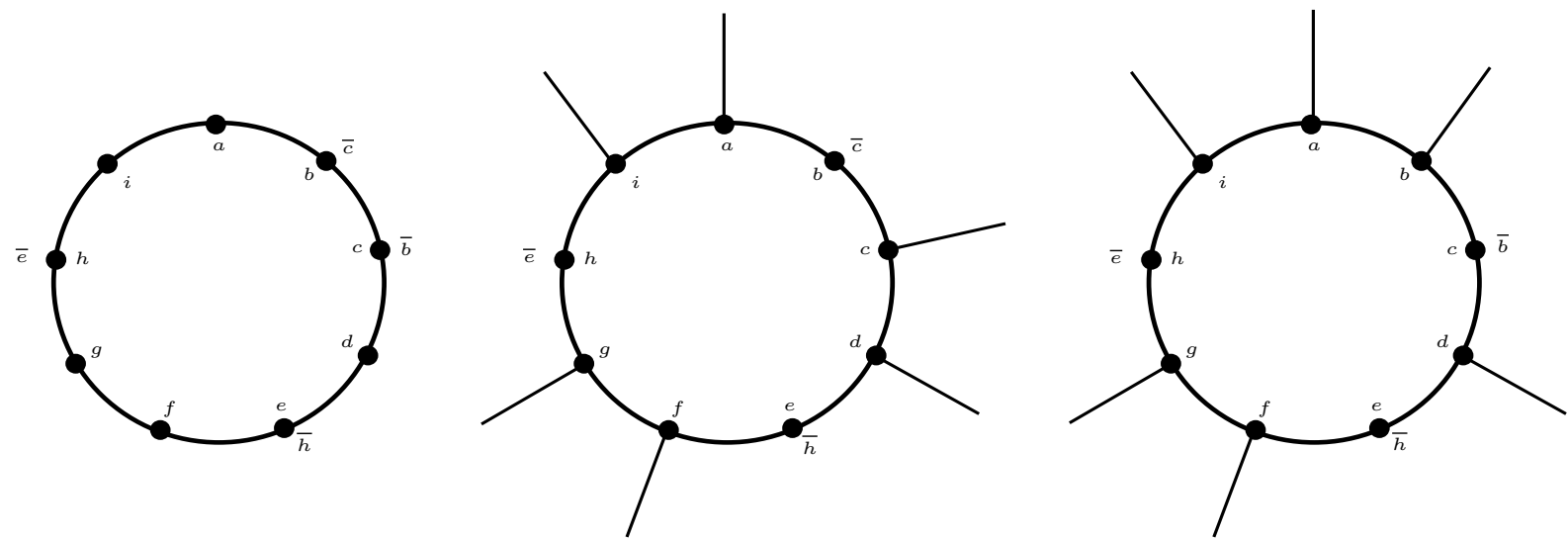

Figure 13: Region $\Delta$

(xii) In this case $\Delta$ is shown in Figure 14. Since $d_{\Delta}\left(v_{b}\right)=d_{\Delta}\left(v_{c}\right)=2$ or $d_{\Delta}\left(v_{c}\right)=$ $d_{\Delta}\left(v_{d}\right)=2$ or $d_{\Delta}\left(v_{d}\right)=d_{\Delta}\left(v_{e}\right)=2$ can not occur therefore $c(\Delta) \leq 0$. 
M. Fazeel Anwar, Mairaj Bibi, M. Saeed Akram / Eur. J. Pure Appl. Math, 12 (2) (2019), 590-604 601

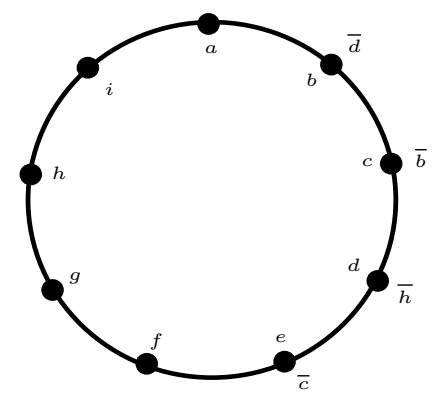

Figure 14: Region $\Delta$

(xiii) In this case $\Delta$ is shown in Figure 15. Since $d_{\Delta}\left(v_{b}\right)=d_{\Delta}\left(v_{c}\right)=2$ or $d_{\Delta}\left(v_{c}\right)=$ $d_{\Delta}\left(v_{d}\right)=2$ can not occur therefore it can be assumed that $d_{\Delta}\left(v_{b}\right)=d_{\Delta}\left(v_{d}\right)=$ $d_{\Delta}\left(v_{h}\right)=2$ as shown in Figure 15. In this case $c(\Delta) \leq 0$.


(xiv) In this case $\Delta$ is shown in Figure 16. Since degree of vertices $v_{d}$ and $v_{e}$ can not be 2 together so there are the following two cases to consider:

(a) $d_{\Delta}\left(v_{b}\right)=d_{\Delta}\left(v_{d}\right)=d_{\Delta}\left(v_{h}\right)=2$;

(b) $d_{\Delta}\left(v_{b}\right)=d_{\Delta}\left(v_{e}\right)=d_{\Delta}\left(v_{h}\right)=2$.

as shown in Figure 16. In both of these cases $c(\Delta) \leq 0$. 

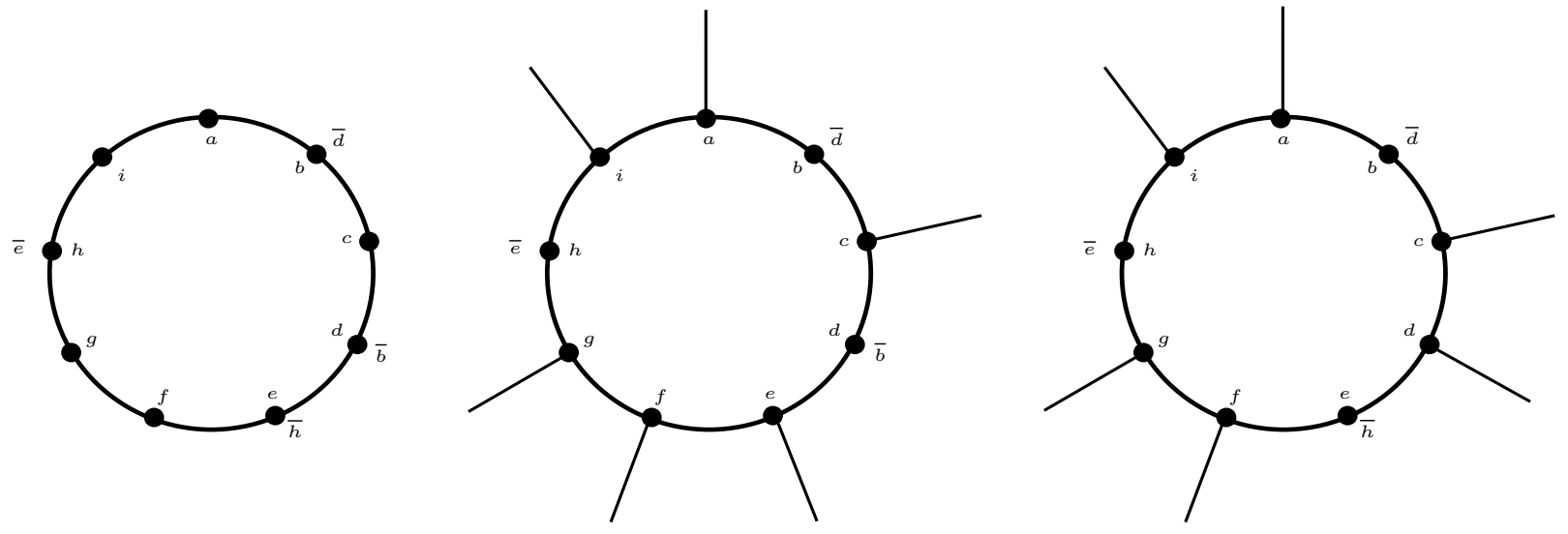

Figure 16: Region $\Delta$

(xv) In this case $\Delta$ is shown in Figure 17. Since degree of vertices $v_{b}$ and $v_{c}$ can not be 2 together so there are the following two cases to consider:

(a) $d_{\Delta}\left(v_{b}\right)=d_{\Delta}\left(v_{e}\right)=d_{\Delta}\left(v_{h}\right)=2$;

(b) $d_{\Delta}\left(v_{c}\right)=d_{\Delta}\left(v_{e}\right)=d_{\Delta}\left(v_{h}\right)=2$.

as shown in Figure 17. In both of these cases $c(\Delta) \leq 0$.
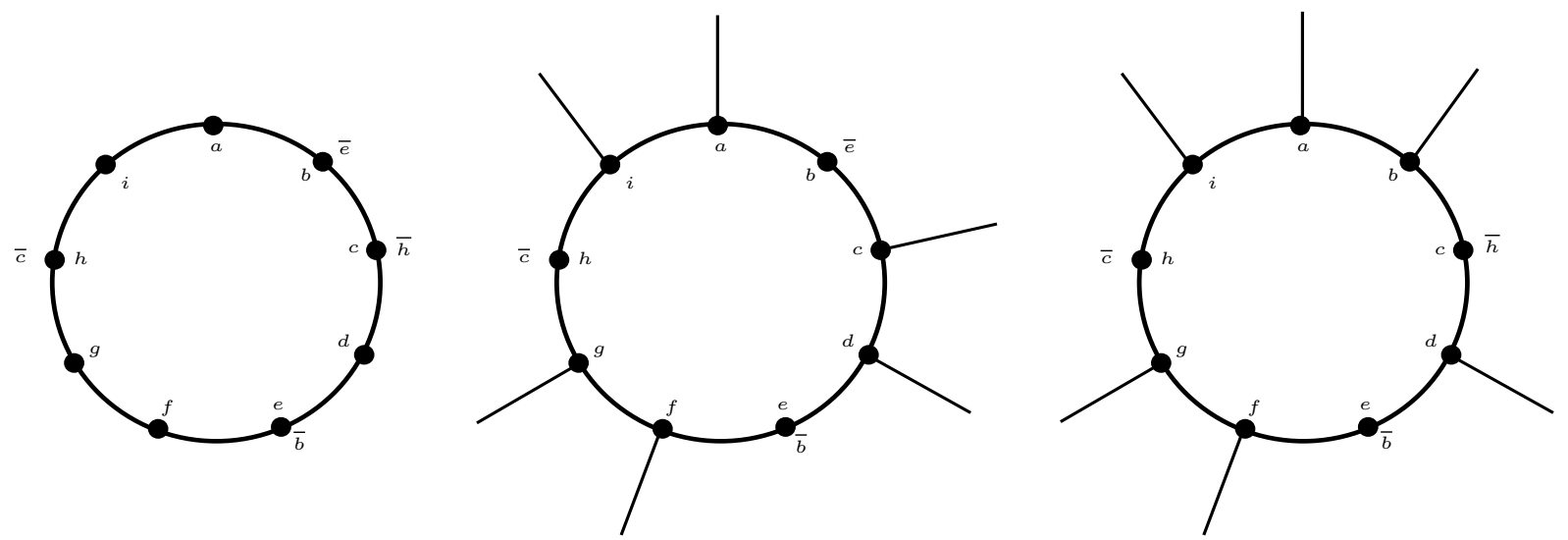

Figure 17: Region $\Delta$

(xvi) In this case $\Delta$ is shown in Figure 18. Since $d_{\Delta}\left(v_{b}\right)=d_{\Delta}\left(v_{c}\right)=2$ or $d_{\Delta}\left(v_{c}\right)=$ $d_{\Delta}\left(v_{d}\right)=2$ can not occur therefore it can be assumed that $d_{\Delta}\left(v_{b}\right)=d_{\Delta}\left(v_{d}\right)=$ $d_{\Delta}\left(v_{h}\right)=2$ as shown in Figure 18. In this case $c(\Delta) \leq 0$. 

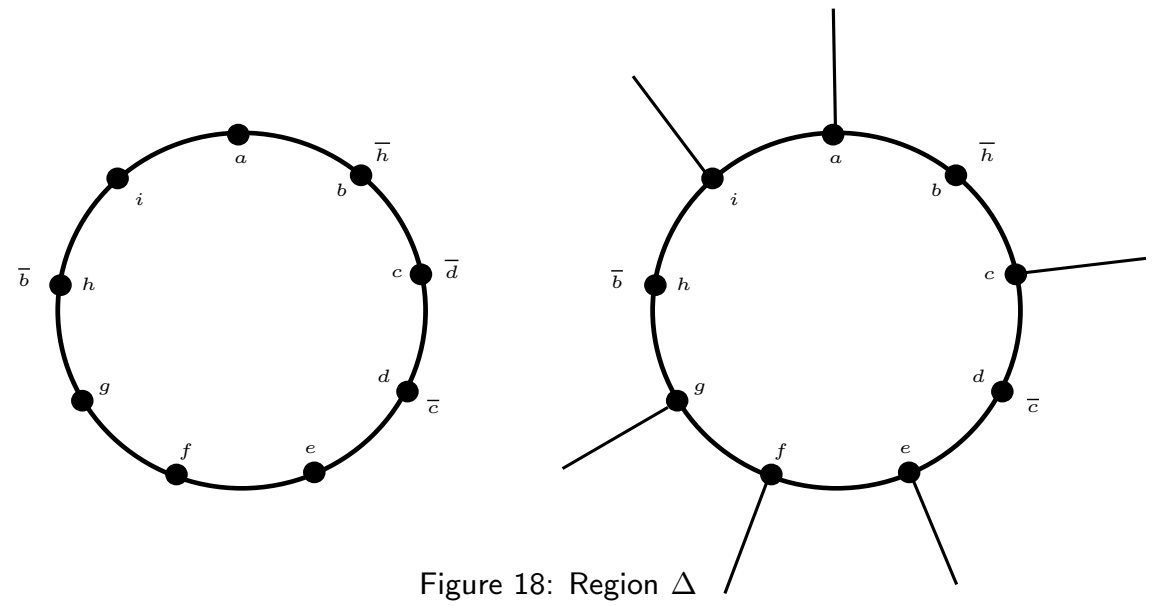

(xvii) In this case $\Delta$ is shown in Figure 19. Since $d_{\Delta}\left(v_{b}\right)=d_{\Delta}\left(v_{c}\right)=2$ or $d_{\Delta}\left(v_{c}\right)=$ $d_{\Delta}\left(v_{d}\right)=2$ or $d_{\Delta}\left(v_{d}\right)=d_{\Delta}\left(v_{e}\right)=2$ can not occur therefore $c(\Delta) \leq 0$.



Figure 19: Region $\Delta$

Remark 1. It is worth mentioning here that a few of the cases still remain open for this equation. These cases are extremely technical in detail and will be considered in a different article.

\section{References}

[1] M F Anwar, M Bibi, and M S Akram. On solvability of certain equations of arbitrary length over torsion-free groups. Preprint, 2019.

[2] M F Anwar, M Bibi, and S Iqbal. On certain equations of arbitrary length over torsion-free groups. Preprint, 2019.

[3] M Bibi. Equations of length seven over torsion free groups. PhD thesis, University of Notingham, 2013. 
[4] M Bibi, M F Anwar, S Iqbal, and M S Akram. Solution of a non-singular equation of length 8 over torsion free groups. Preprint, 2019.

[5] M Bibi and M Edjvet. Solving equations of length seven over torsion-free groups. Journal of Group Theory, 21(1):147-164, 2018.

[6] W A Bogley and S J Pride. Aspherical relative presentations. Proceedings of the Edinburgh Mathematical Society, 35, 1992.

[7] S D Brodski and J Howie. One-relator products of torsion-free groups. Glasgow Mathematical Journal, 35(1):99-104, 1993.

[8] J Howie. The solution of length three equations over groups. Proceedings of the Edinburgh Mathematical Society, 26(2):89-96, 1983.

[9] S V Ivanov and A A Klyachko. Solving equations of length at most six over torsionfree groups. Journal od Group Theory, 3(3):329-337, 2000.

[10] S K Kim. On the asphericity of length-6 relative presentations with torsion-free coefficients. Proceedings of the Edinburgh Mathematical Society, 51(1):201-214, 2008.

[11] F Levin. Solutions of equations over groups. Bulletin of American Mathematical Society, 68, 1962.

[12] M I Prishchepov. On small length equations over torsion-free groups. International Journal of Algebra and Computation, 4(4):575-589, 1994. 